\title{
Set of new observables in the process $e^{+} e^{-} \rightarrow \mathrm{ZHH}$
}

\author{
Junya Nakamura* \\ Institut für Theoretische Physik, Universität Tübingen, 72076 Tübingen, Germany
}

(Received 27 October 2017; published 29 January 2018)

\begin{abstract}
Consequences of nonstandard Higgs couplings in the final-state distributions of the process $e^{+} e^{-} \rightarrow$ $\mathrm{ZHH}$ are studied. We derive an analytic expression for the differential cross section, which has in the most general case nine nonzero functions. These functions are the coefficients of nine angular terms, depend on the Higgs couplings, and can be experimentally measured as observables. Symmetry properties of these nine functions are carefully discussed, and they are divided into four categories under $C P$ and $C P \tilde{T}$. The relations between our observables and the observables which exist in the literature are also clarified. We numerically study the dependence of our observables on the parameters in an effective Lagrangian for the Higgs couplings. It is shown that these new observables depend on most of the effective Lagrangian parameters in different ways from the total cross section. A benefit from longitudinally polarized $e^{+} e^{-}$ beams is also discussed.
\end{abstract}

DOI: 10.1103/PhysRevD.97.013005

\section{INTRODUCTION}

One of the main targets of experiments at future $e^{+} e^{-}$ colliders is the measurement of the trilinear self-coupling $\lambda_{H}$ of the Higgs boson [1-5]. The process $e^{+} e^{-} \rightarrow Z H H$ is expected to be the best reaction to measure $\lambda_{H}$ in the earlier stage of experiments [6-16] (i.e. the center-of-mass energy $\sqrt{s} \simeq 500 \mathrm{GeV}$ ) for the discovered Higgs boson with mass $\simeq 125 \mathrm{GeV}[17,18]$. The process is sensitive to the couplings $H H Z Z$ [19] and $H H Z \gamma$, too, which cannot be accessed through single Higgs boson production processes such as $e^{+} e^{-} \rightarrow Z H$.

Because of its importance, many authors have investigated the process. The total cross section in the standard model (SM) was calculated for the first time in Ref. [19]. This work was followed by several studies [20,21]. These papers numerically calculated various distributions of the final particles, too. The one-loop radiative corrections to the process were calculated in Refs. [22,23]. The total cross section in the minimal supersymmetric extension of the SM [24-28], that in composite Higgs models [29,30], and that in other several new physics models [31] have also been studied in detail. References [25-27] included the analytic form of the two Higgs energy distributions. The accuracy of measuring $\lambda_{H}$ through the process $e^{+} e^{-} \rightarrow \mathrm{ZHH}$ at future $e^{+} e^{-}$colliders has been studied in Refs. [6-16] by

\footnotetext{
*junya.nakamura@itp.uni-tuebingen.de
}

Published by the American Physical Society under the terms of the Creative Commons Attribution 4.0 International license. Further distribution of this work must maintain attribution to the author(s) and the published article's title, journal citation, and DOI. Funded by SCOAP. assuming that all the other couplings are the SM values. The expected constraints on several parameters (including parameters which affect $\lambda_{H}$ ) in an effective Lagrangian have been discussed in Refs. [32-35]. Reference [33] included the analytic form for the invariant mass distribution of the two Higgs bosons.

Most of the above studies, however, restricted themselves to the total cross section as input from the experiments. This will not be a problem, if one intends to determine only one parameter such as $\lambda_{H}$. However, if one intends to determine more than one parameter at the same time as studied in Refs. [32-35], measuring only the total cross section is not enough and one needs to consider other observables such as the invariant mass distribution of the two Higgs bosons [33,34]. The purpose of this paper is to introduce such observables in a rather different way. We find nine observables as the coefficients of nine angular terms in the differential cross section, one of which is directly related to the total cross section. The other eight observables have not been studied in the literature. Symmetry properties of the nine observables are clarified, and they are divided into four categories under $C P$ and $C P \tilde{T}$ [36]: four even-even, one even-odd, two odd-even, and two odd-odd. The $C P$-odd observables directly measure $C P$ nonconservation and the $C P \tilde{T}$-odd observables rescattering effects. To our knowledge, any $C P$-odd and/or $C P \tilde{T}$-odd observables in this process have not been constructed so far.

This paper is organized as follows. In Sec. II, we explain kinematics of the process $e^{+} e^{-} \rightarrow Z H H$. In Sec. III, we give an analytic expression for the differential cross section. The differential cross section has nine nonzero functions in the most general case, and these nine functions can be 
measured experimentally. We rederive the analytic forms of the observables which exist in the literature and have been widely used, such as the invariant mass distribution of the two Higgs bosons. We show that all of these observables are directly related to just one of our nine functions. The analytic form of the $Z$ boson polar angle distribution is also derived. In Sec. IV, the symmetry properties of the nine functions are studied. In Sec. V, we form observables in terms of our nine functions and numerically study the dependence of these observables on the parameters in an effective Lagrangian for the Higgs couplings. We show that our new observables depend on most of the effective Lagrangian parameters in different ways than the total cross section. It is also shown that the sensitivity of the $C P \tilde{T}$-odd observable can be significantly enhanced by means of longitudinally polarized $e^{+} e^{-}$beams. Section VI summarizes our findings.

\section{KINEMATICS}

Our coordinate system in the center-of-mass (c.m.) frame of the colliding $e^{+} e^{-}$beams is described in Fig. 1. ${ }^{1}$ The four-momentum and helicity of each particle are shown in parentheses. A single object as the sum of the two Higgs bosons is represented by $2 H$, whose fourmomentum is $q^{\mu}$. We choose the direction of $\vec{q}$ as the $z$ axis and the $\vec{p}_{1} \times \vec{k}$ direction as the $y$ axis. The scattering $p_{1}+p_{2} \rightarrow k+q$ takes place in the $x-z$ plane. The polar angle of the $Z$ boson from the electron momentum direction is denoted by $\Theta$. Because we neglect the $e^{ \pm}$masses and $e^{-}$ and $e^{+}$always construct a four-vector in our process, the helicity of $e^{+}$is always opposite to that of $e^{-}$. In this coordinate system, the four-momenta can be parametrized as follows:

$$
\begin{aligned}
p^{\mu} & \equiv\left(p_{1}+p_{2}\right)^{\mu}=(E, 0,0,0), \\
p_{1}^{\mu} & =\frac{E}{2}(1, \sin \Theta, 0,-\cos \Theta), \\
p_{2}^{\mu} & =\frac{E}{2}(1,-\sin \Theta, 0, \cos \Theta), \\
k^{\mu} & =(w, 0,0,-l), \\
q^{\mu} & =\left(q_{1}+q_{2}\right)^{\mu}=(E-w, 0,0, l),
\end{aligned}
$$

where $E$ is the $e^{+} e^{-}$c.m. energy, $w$ is the energy of the $Z$ boson: $w=\left(E^{2}+m_{Z}^{2}-Q^{2}\right) /(2 E)$ where $Q^{2}=q \cdot q,-l$ is the momentum of the $Z$ boson: $l=\sqrt{w^{2}-m_{Z}^{2}}$, and $q_{1,2}^{\mu}$ are the four-momenta of the two Higgs bosons. We parametrize $q_{1,2}^{\mu}$ in the rest frame of $q^{\mu}$ as

$$
\begin{aligned}
q^{\mu} & =\left(q_{1}+q_{2}\right)^{\mu}=(Q, 0,0,0), \\
q_{1}^{\mu} & =(Q / 2, r \sin \xi \cos \phi, r \sin \xi \sin \phi, r \cos \xi), \\
q_{2}^{\mu} & =(Q / 2,-r \sin \xi \cos \phi,-r \sin \xi \sin \phi,-r \cos \xi),
\end{aligned}
$$

where $r=\sqrt{Q^{2} / 4-m_{H}^{2}}$. Since we cannot distinguish the two Higgs bosons, we define the regions of the angles as $0 \leq \xi \leq \pi / 2$ and $0 \leq \phi \leq 2 \pi$ and identify the Higgs boson whose momentum along the $z$ axis is positive as the Higgs boson that has $q_{1}^{\mu}$. The four-momenta $q_{1,2}^{\mu}$ in our $e^{+} e^{-}$c.m. frame can easily be obtained by a single boost along the positive direction of the $z$ axis,

$$
\begin{aligned}
& q_{1}^{\mu}=\left(\frac{E-w}{2}+\frac{l}{Q} r \cos \xi, r \sin \xi \cos \phi, r \sin \xi \sin \phi, \frac{l}{2}+\frac{E-w}{Q} r \cos \xi\right), \\
& q_{2}^{\mu}=\left(\frac{E-w}{2}-\frac{l}{Q} r \cos \xi,-r \sin \xi \cos \phi,-r \sin \xi \sin \phi, \frac{l}{2}-\frac{E-w}{Q} r \cos \xi\right) .
\end{aligned}
$$

We introduce the four-momenta $t^{\mu}$ and $u^{\mu}$ of the intermediate $Z$ boson or the photon $A$ in the diagrams (2) and (3) of Fig. 2, respectively,

$$
\begin{aligned}
t^{\mu} & =\left(k+q_{1}\right)^{\mu} \\
& =\left(\frac{E+w}{2}+\frac{l}{Q} r \cos \xi, r \sin \xi \cos \phi, r \sin \xi \sin \phi,-\frac{l}{2}+\frac{E-w}{Q} r \cos \xi\right), \\
u^{\mu} & =\left(k+q_{2}\right)^{\mu} \\
& =\left(\frac{E+w}{2}-\frac{l}{Q} r \cos \xi,-r \sin \xi \cos \phi,-r \sin \xi \sin \phi,-\frac{l}{2}-\frac{E-w}{Q} r \cos \xi\right) .
\end{aligned}
$$

\footnotetext{
${ }^{1}$ Figures 1, 2, 3, 4 are drawn by using the program JaxoDraw [37].
} 


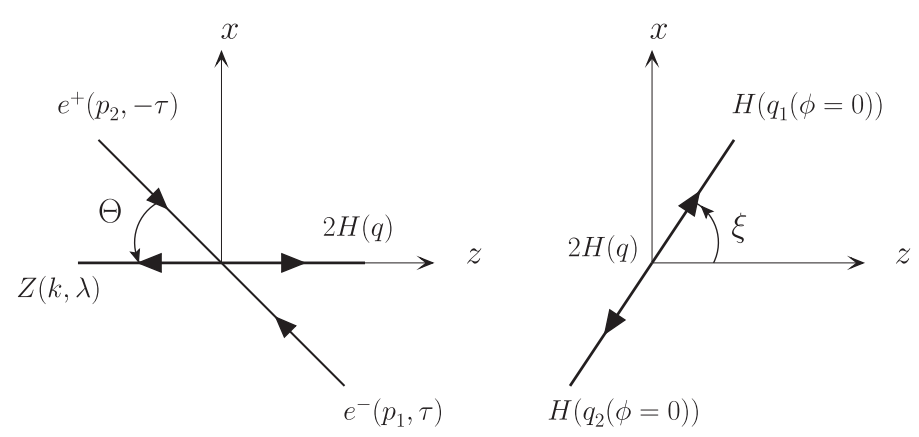

FIG. 1. Left: The coordinate system in the c.m. frame of the colliding $e^{+} e^{-}$beams. The four-momentum and helicity of each particle are shown in parentheses. A single object as the sum of the two Higgs bosons is represented by $2 H$, whose four-momentum is $q^{\mu}$. The direction of $\vec{q}$ is chosen as the $z$ axis and the $\vec{p}_{1} \times \vec{k}$ direction as the $y$ axis. Right: The coordinate system in the c.m. frame of the two Higgs bosons (i.e. the rest frame of $q^{\mu}$ ). The parametrization of the four-momenta in this frame is given in Eq. (2.2).

\section{DIFFERENTIAL CROSS SECTION}

We present an analytic expression for the differential cross section by assuming nonstandard Higgs couplings. The effective Lagrangian, from which we obtain the Higgs couplings, will be given in Sec. V. The Feynman diagrams contributing to the scattering amplitudes of the process $e^{+} e^{-} \rightarrow \mathrm{ZHH}$ with our nonstandard Higgs couplings are shown in Fig. 2. It is an easy task to derive the scattering amplitudes in terms of the kinematic variables defined in Sec. II. We find that the amplitude-squared summed over the $Z$ boson helicity $\lambda$ for a given electron helicity $\tau$ has the following form in the most general case:

$$
\begin{aligned}
\sum_{\lambda= \pm, 0}\left|\mathcal{M}_{\tau}^{\lambda}\right|^{2}= & F_{1}\left(1+\cos ^{2} \Theta\right)+F_{2}\left(1-3 \cos ^{2} \Theta\right)+F_{3} \cos \Theta \\
& +F_{4} \sin \Theta \cos \phi+F_{5} \sin 2 \Theta \cos \phi \\
& +F_{6} \sin ^{2} \Theta \cos 2 \phi+F_{7} \sin \Theta \sin \phi \\
& +F_{8} \sin 2 \Theta \sin \phi+F_{9} \sin ^{2} \Theta \sin 2 \phi
\end{aligned}
$$

where the $\Theta$ and $\phi$ dependences are completely factorized and the nine functions $F_{i}$ as the angular coefficients are independent of these two angles: $F_{i}=F_{i}(\tau, Q, \xi){ }^{2}$ The nine functions $F_{i}$ depend on the Higgs couplings. The explicit expressions of $F_{i}$ are provided in the Appendix in terms of effective Lagrangian parameters given in Sec. V. The nine functions $F_{i}$ can be experimentally determined by measuring the angles $\Theta$ and $\phi$, and therefore used to study the Higgs couplings. For an approach to isolate the functions, see Eqs. (3.28) and (3.29) of Ref. [38]. By means of Eq. (3.1), the complete differential cross section for a given electron helicity $\tau$ is given by

\footnotetext{
${ }^{2}$ The functions $F_{i}$ depend on the $e^{+} e^{-}$c.m. energy (i.e. $E$ in our notation); hence $F_{i}=F_{i}(\tau, Q, \xi, E)$ is the more appropriate expression. However, we regard $E$ as a fixed value and do not write $E$ explicitly in the arguments of functions throughout the paper.
}

$$
\begin{aligned}
\frac{d \sigma(\tau)}{d \Omega} & \equiv \frac{d \sigma(\tau)}{d \cos \Theta d Q^{2} d \cos \xi d \phi} \\
& =\frac{1}{1024 \pi^{4}} \frac{l}{E^{3}} \sqrt{1-\frac{4 m_{H}^{2}}{Q^{2}}} \sum_{\lambda= \pm, 0}\left|\mathcal{M}_{\tau}^{\lambda}\right|^{2} .
\end{aligned}
$$

By performing the integration over $\phi$, we obtain the analytic form of the $\cos \Theta$ distribution,

$$
\begin{aligned}
\int_{0}^{2 \pi} d \phi \frac{d \sigma(\tau)}{d \Omega}= & \frac{1}{512 \pi^{3}} \frac{l}{E^{3}} \sqrt{1-\frac{4 m_{H}^{2}}{Q^{2}}}\left[F_{1}\left(1+\cos ^{2} \Theta\right)\right. \\
& \left.+F_{2}\left(1-3 \cos ^{2} \Theta\right)+F_{3} \cos \Theta\right],
\end{aligned}
$$

where the other six terms were eliminated by the integration. The numerical studies of the $\cos \Theta$ distribution in the literature (e.g. $[8,34])$ actually probe the three coefficients, which can be obtained by integrating Eq. (3.3) over $Q^{2}$ and $\cos \xi$,

$$
\begin{aligned}
\frac{d \sigma(\tau)}{d \cos \Theta}= & \int_{4 m_{H}^{2}}^{\left(E-m_{Z}\right)^{2}} d Q^{2} \int_{0}^{1} d \cos \xi \int_{0}^{2 \pi} d \phi \frac{d \sigma(\tau)}{d \Omega} \\
= & \int_{4 m_{H}^{2}}^{\left(E-m_{Z}\right)^{2}} d Q^{2} \int_{0}^{1} d \cos \xi \frac{1}{512 \pi^{3}} \frac{l}{E^{3}} \sqrt{1-\frac{4 m_{H}^{2}}{Q^{2}}} \\
& \times\left[F_{1}\left(1+\cos ^{2} \Theta\right)+F_{2}\left(1-3 \cos ^{2} \Theta\right)+F_{3} \cos \Theta\right] .
\end{aligned}
$$

By further integrating Eq. (3.3) over $\cos \Theta$, we obtain

$$
\begin{aligned}
\frac{d \sigma(\tau)}{d Q^{2} d \cos \xi} & =\int_{-1}^{1} d \cos \Theta \int_{0}^{2 \pi} d \phi \frac{d \sigma(\tau)}{d \Omega} \\
& =\frac{1}{192 \pi^{3}} \frac{l}{E^{3}} \sqrt{1-\frac{4 m_{H}^{2}}{Q^{2}}} F_{1}
\end{aligned}
$$

where the other two terms were eliminated by the integration. From this, we can easily obtain the analytic form of 


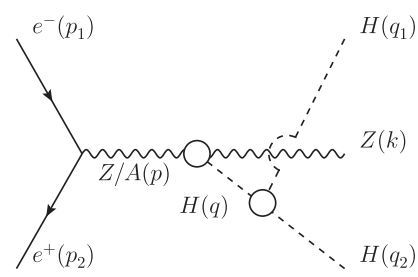

(1)

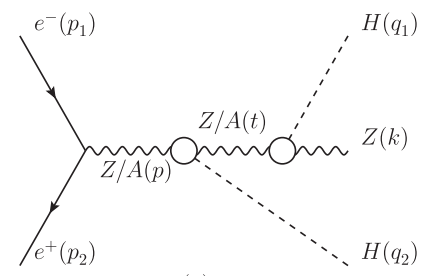

(2)

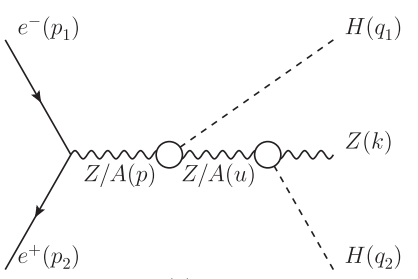

(3)

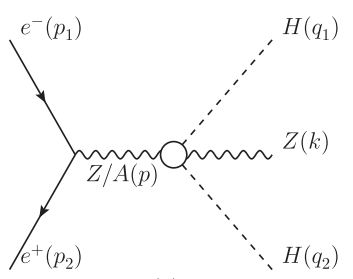

(4)

FIG. 2. Feynman diagrams for $e^{+} e^{-} \rightarrow Z H H$ with our effective Lagrangian Eq. (5.1). The four-momentum of each particle is shown in parentheses.

the $Q^{2}$ distribution which has been numerically studied e.g. in Refs. [9,14] and that of the $\cos \xi$ distribution which Ref. [7] mentions can be a good observable for measuring $\lambda_{H}$,

$$
\frac{d \sigma(\tau)}{d Q^{2}}=\int_{0}^{1} d \cos \xi \frac{1}{192 \pi^{3}} \frac{l}{E^{3}} \sqrt{1-\frac{4 m_{H}^{2}}{Q^{2}}} F_{1},
$$

$\frac{d \sigma(\tau)}{d \cos \xi}=\int_{4 m_{H}^{2}}^{\left(E-m_{Z}\right)^{2}} d Q^{2} \frac{1}{192 \pi^{3}} \frac{l}{E^{3}} \sqrt{1-\frac{4 m_{H}^{2}}{Q^{2}}} F_{1}$.

The total cross section for a given electron helicity $\tau$ is given by

$\sigma(\tau)=\int_{4 m_{H}^{2}}^{\left(E-m_{Z}\right)^{2}} d Q^{2} \int_{0}^{1} d \cos \xi \frac{1}{192 \pi^{3}} \frac{l}{E^{3}} \sqrt{1-\frac{4 m_{H}^{2}}{Q^{2}}} F_{1}$.

We introduce the scaling variables

$$
x_{1}=\frac{2 q_{1}^{0}}{E}, \quad x_{2}=\frac{2 q_{2}^{0}}{E} .
$$

Note that $q_{1}^{0}$ and $q_{2}^{0}$ are defined in Eq. (2.3). By straightforward variable conversions in Eq. (3.5), we obtain

$$
\frac{d \sigma(\tau)}{d x_{1} d x_{2}}=\frac{1}{192 \pi^{3}} F_{1},
$$

which has been derived in Refs. [25-27], and

$$
\frac{d \sigma(\tau)}{d Q^{2} d(t \cdot t)}=\frac{1}{192 \pi^{3} E^{4}} F_{1},
$$

which has been derived in Ref. [33]. Note that the phase space region $0 \leq \xi \leq \pi / 2$ [see below Eq. (2.2)] corresponds to $x_{1} \geq x_{2}$ and $t \cdot t \geq u \cdot u$. The four-momenta $t^{\mu}$ and $u^{\mu}$ are defined in Eq. (2.4). We emphasize that the observables that exist in the literature and are rederived above in Eqs. (3.6), (3.7), (3.9), and (3.10) are directly related to the function $F_{1}$, which is just one of the nine functions in the differential cross section.

\section{SYMMETRY PROPERTIES}

The conditions imposed by symmetries lead to constraints on some of the nine functions $F_{i}$. The picture in Fig. 3 shows the original states and the states after the charge-conjugation $(C)$ and parity $(P)$ transformation. After the $C P$ transformation, the states are simply rotated around the $y$ axis by $\pi$, and we make the four-momentum $q^{\mu}$ come back to the original position. Note that we always have a freedom of performing three-dimensional spatial rotations. While $q^{\mu}$ is unchanged, the four-momenta $q_{1}^{\mu}$ and $q_{2}^{\mu}$ are changed by the $C P$ transformation and the rotation as

$$
\begin{aligned}
q_{1}^{\mu} & =\left(\frac{E-w}{2}+\frac{l}{Q} r \cos \xi, r \sin \xi \cos \phi, r \sin \xi \sin \phi, \frac{l}{2}+\frac{E-w}{Q} r \cos \xi\right), \\
& \underset{C P}{\longrightarrow}\left(\frac{E-w}{2}+\frac{l}{Q} r \cos \xi,-r \sin \xi \cos \phi,-r \sin \xi \sin \phi,-\frac{l}{2}-\frac{E-w}{Q} r \cos \xi\right), \\
& \underset{\text { Rotation }}{\longrightarrow}\left(\frac{E-w}{2}+\frac{l}{Q} r \cos \xi, r \sin \xi \cos \phi,-r \sin \xi \sin \phi, \frac{l}{2}+\frac{E-w}{Q} r \cos \xi\right) .
\end{aligned}
$$

Notice that only the $y$ component of $q_{1}^{\mu}$ changes sign, which indicates that the azimuthal angle $\phi$ is $2 \pi-\phi$ after the transformations. Therefore, $C P$ invariance leads to the following relation for the differential cross section:

$$
d \sigma(\tau, \Theta, Q, \xi, \phi)=d \sigma(\tau, \Theta+\pi, Q, \xi, 2 \pi-\phi),
$$

where the one on the left-hand side corresponds to the original states shown in the left picture of Fig. 3 and the one on the right-hand side corresponds to the states after the $C P$ 


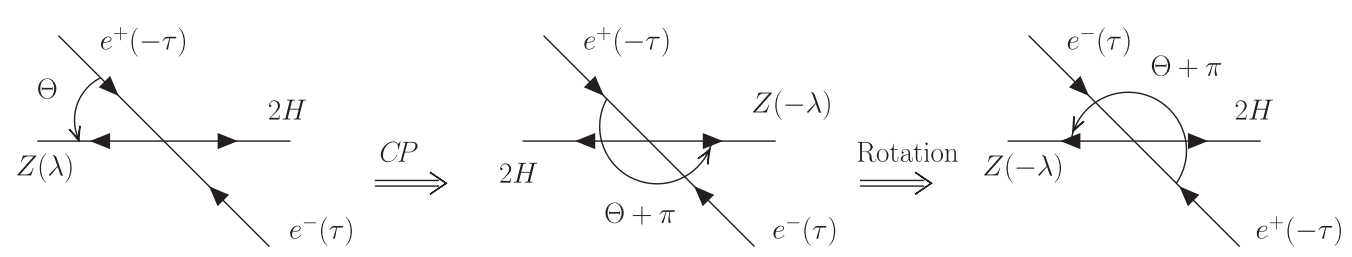

FIG. 3. The states after the $C P$ transformation are shown. At the second step, a rotation around the $y$ axis by $\pi$ is performed. The helicity of each particle is shown in parentheses.
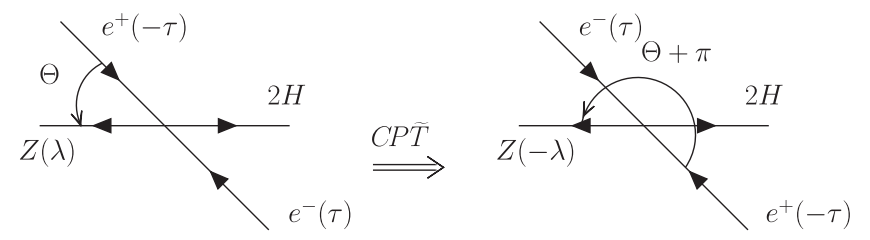

FIG. 4. The states after the $C P$ and time-reversal transformation without interchanging the initial and final states $(\tilde{T})$ are shown. The helicity of each particle is shown in parentheses.

transformation and the rotation shown in the right picture of Fig. 3. The explicit form of the right-hand side of this equation is, from Eqs. (3.1) and (3.2), given by

$$
\begin{aligned}
\frac{d \sigma(\tau)}{d \cos \Theta d Q^{2} d \cos \xi d \phi} \\
=\frac{1}{1024 \pi^{4}} \frac{l}{E^{3}} \sqrt{1-\frac{4 m_{H}^{2}}{Q^{2}}}\left[F_{1}\left(1+\cos ^{2} \Theta\right)\right. \\
\quad+F_{2}\left(1-3 \cos ^{2} \Theta\right)-F_{3} \cos \Theta-F_{4} \sin \Theta \cos \phi \\
\quad+F_{5} \sin 2 \Theta \cos \phi+F_{6} \sin ^{2} \Theta \cos 2 \phi+F_{7} \sin \Theta \sin \phi \\
\left.\quad-F_{8} \sin 2 \Theta \sin \phi-F_{9} \sin ^{2} \Theta \sin 2 \phi\right] .
\end{aligned}
$$

Let us remind the reader that $F_{i}$ are independent of $\Theta$ and $\phi$; thus they have the same forms in both sides of Eq. (4.2). ${ }^{3}$ In Eq. (4.3), we observe that the four terms of the nine terms change sign. These terms, namely the $F_{3}, F_{4}, F_{8}$, and $F_{9}$ terms, are $C P$-odd. The four functions $F_{3}, F_{4}, F_{8}$, and $F_{9}$ must be zero if $C P$ is conserved. In other words, observation of nonzero values in these four functions signals $C P$ nonconservation.

Second, the picture in Fig. 4 shows the original states and the states after the $C, P$, and time-reversal transformation without interchanging the initial and final states (i.e. it does not reverse the time flow from the initial state to the final state). We denote it by $\tilde{T}$. $C P \tilde{T}$ invariance leads to the following relation for the differential cross section:

$$
d \sigma(\tau, \Theta, Q, \xi, \phi)=d \sigma(\tau, \Theta+\pi, Q, \xi, \phi),
$$

\footnotetext{
${ }^{3}$ We actually perform the rotation in order to make all of $F_{i}$ invariant. Some of $F_{i}$ are not invariant without the rotation. Even without the rotation, however, our conclusion that the $F_{3}, F_{4}, F_{8}$, and $F_{9}$ terms are $C P$-odd should remain the same, as long as physics is invariant under three-dimensional spatial rotations.
}

where the one on the left-hand side corresponds to the original states shown in the left picture of Fig. 4 and the one on the right-hand side corresponds to the states after the $C P \tilde{T}$ transformation shown in the right picture of Fig. 4. The explicit form of the right-hand side of this equation is, from Eqs. (3.1) and (3.2), given by

$$
\begin{aligned}
\frac{d \sigma(\tau)}{d \cos \Theta d Q^{2} d \cos \xi d \phi} \\
=\frac{1}{1024 \pi^{4}} \frac{l}{E^{3}} \sqrt{1-\frac{4 m_{H}^{2}}{Q^{2}}} \\
\quad \times\left[F_{1}\left(1+\cos ^{2} \Theta\right)+F_{2}\left(1-3 \cos ^{2} \Theta\right)-F_{3} \cos \Theta\right. \\
\quad-F_{4} \sin \Theta \cos \phi+F_{5} \sin 2 \Theta \cos \phi+F_{6} \sin ^{2} \Theta \cos 2 \phi \\
\left.-F_{7} \sin \Theta \sin \phi+F_{8} \sin 2 \Theta \sin \phi+F_{9} \sin ^{2} \Theta \sin 2 \phi\right],
\end{aligned}
$$

where we observe that the three terms of the nine terms change sign. These terms, namely the $F_{3}, F_{4}$, and $F_{7}$ terms, are $C P \tilde{T}$-odd. The three functions $F_{3}, F_{4}$, and $F_{7}$ must be zero if $C P \tilde{T}$ is conserved. In other words, observation of nonzero values in these three functions signals $C P \tilde{T}$ violation, which indicates the existence of rescattering effects [36]. In Table I, we summarize the symmetry properties of the functions. Notice that $F_{3}$ and $F_{4}$ are both

TABLE I. Symmetry properties of the nine functions in the differential cross section. The symbol $+(-)$ means that the function is even (odd) under $C P$ or $C P \tilde{T}$. Observation of nonzero values in the $C P$-odd functions signals $C P$ nonconservation. Observation of nonzero values in the $C P \tilde{T}$-odd functions indicates the existence of rescattering effects. The symbol $\circ$ in the last column indicates that the function can be suppressed without polarized $e^{+} e^{-}$beams.

\begin{tabular}{lccc}
\hline \hline & \multicolumn{2}{c}{ Symm. properties } & \\
\cline { 2 - 3 } Functions & $C P$ & $C P \tilde{T}$ & Beam pol. \\
\hline$F_{1}$ & + & + & - \\
$F_{2}$ & + & + & - \\
$F_{3}$ & - & - & $\circ$ \\
$F_{4}$ & - & - & $\circ$ \\
$F_{5}$ & + & + & - \\
$F_{6}$ & + & + & - \\
$F_{7}$ & + & - & $\circ$ \\
$F_{8}$ & - & + & - \\
$F_{9}$ & - & + & - \\
\hline \hline
\end{tabular}


$C P$-odd and $C P \tilde{T}$-odd. Once we experimentally confirm that both the $C P$-odd functions (i.e. $F_{8}$ and $F_{9}$ ) and the $C P \tilde{T}$-odd function (i.e. $F_{7}$ ) are small, we may ignore $F_{3}$ and $F_{4}$ since these are doubly suppressed.

\section{NUMERICAL STUDIES}

We obtain nonstandard Higgs couplings to the Higgs boson itself, the $Z$ boson, and the photon from the following effective Lagrangian [39]:

$$
\begin{aligned}
\mathcal{L}_{\text {eff }}= & \left(1+\delta_{1}\right) m_{Z}^{2} \frac{H}{v} Z_{\mu} Z^{\mu}+\sum_{V=Z, A}\left\{\delta_{2}^{V} \frac{H}{v} Z_{\mu \nu} V^{\mu \nu}+\delta_{3}^{V} \frac{1}{v}\left[\left(\partial^{\mu} H\right) Z^{\nu}-\left(\partial^{\nu} H\right) Z^{\mu}\right] V_{\mu \nu}+\tilde{\delta}_{4}^{V} \frac{H}{v} Z_{\mu \nu} \tilde{V}^{\mu \nu}\right\} \\
& +\left(1+\delta_{5}\right) m_{Z}^{2} \frac{H^{2}}{2 v^{2}} Z_{\mu} Z^{\mu}+\sum_{V=Z, A}\left\{\delta_{6}^{V} \frac{H^{2}}{2 v^{2}} Z_{\mu \nu} V^{\mu \nu}+\delta_{7}^{V} \frac{H}{v^{2}}\left[\left(\partial^{\mu} H\right) Z^{\nu}-\left(\partial^{\nu} H\right) Z^{\mu}\right] V_{\mu \nu}+\tilde{\delta}_{8}^{V} \frac{H^{2}}{2 v^{2}} Z_{\mu \nu} \tilde{V}^{\mu \nu}\right\} \\
& +\delta_{2}^{A A} \frac{H}{v} A_{\mu \nu} A^{\mu \nu}+\tilde{\delta}_{4}^{A A} \frac{H}{v} A_{\mu \nu} \tilde{A}^{\mu \nu}-\frac{m_{H}^{2}}{2 v}\left(1+\delta_{9}\right) H^{3}+\delta_{10} \frac{H}{v}\left(\partial^{\mu} H\right)^{2},
\end{aligned}
$$

where $Z_{\mu \nu}=\partial_{\mu} Z_{\nu}-\partial_{\nu} Z_{\mu}, A_{\mu \nu}=\partial_{\mu} A_{\nu}-\partial_{\nu} A_{\mu}, Z_{\mu}$ is the $Z$ boson field, $A_{\mu}$ is the photon field, $\tilde{Z}^{\mu \nu}=\frac{1}{2} \epsilon^{\mu \nu \rho \sigma} Z_{\rho \sigma}$ and $\tilde{A}^{\mu \nu}=\frac{1}{2} \epsilon^{\mu \nu \rho \sigma} A_{\rho \sigma}$ with our convention $\epsilon_{0123}=+1$, and $v$ is the vacuum expectation value of the Higgs doublet field: $v^{-2}=\sqrt{2} G_{F}$. All of the 18 coefficients $\delta_{i}$ are zero at the tree level in the SM. The five operators whose coefficients are $\tilde{\delta}_{4}^{V}, \tilde{\delta}_{8}^{V}$, and $\tilde{\delta}_{4}^{A A}$ are $C P$-odd, and the other 13 operators are $C P$-even. If both the $C P$-even operator(s) and the $C P$-odd operator(s) exist, the theory is not $C P$ conserving. If we consider the $S U(2) \times U(1)$ gauge invariant dimension six operators which consist of the gauge boson fields and the Higgs doublet field, there are eight $C P$-even operators and five $C P$-odd operators $[39,40]$ which contribute to the Higgs couplings relevant to our process and their effects can be expressed as their contributions to our 18 coefficients $\delta_{i}$. In addition, two $C P$-even operators affect our process through the renormalization of the SM parameters and the external $Z$ and Higgs fields [39]. Feynman diagrams for the process $e^{+} e^{-} \rightarrow Z H H$ with this effective Lagrangian have already been shown in Fig. 2. We have 12 diagrams maximum. The nine functions $F_{i}$ in Eq. (3.1) in terms of $\delta_{i}$ are provided in the Appendix.

We integrate the differential cross section in Eq. (3.2) over $Q^{2}$ and $\cos \xi$,

$$
\begin{aligned}
\frac{d \sigma(\tau)}{d \cos \Theta d \phi}= & \mathcal{F}_{1}\left(1+\cos ^{2} \Theta\right)+\mathcal{F}_{2}\left(1-3 \cos ^{2} \Theta\right)+\mathcal{F}_{3} \cos \Theta \\
& +\mathcal{F}_{4} \sin \Theta \cos \phi+\mathcal{F}_{5} \sin 2 \Theta \cos \phi \\
& +\mathcal{F}_{6} \sin ^{2} \Theta \cos 2 \phi+\mathcal{F}_{7} \sin \Theta \sin \phi \\
& +\mathcal{F}_{8} \sin 2 \Theta \sin \phi+\mathcal{F}_{9} \sin ^{2} \Theta \sin 2 \phi
\end{aligned}
$$

where

$$
\begin{aligned}
\mathcal{F}_{i}(\tau)= & \int_{4 m_{H}^{2}}^{\left(E-m_{Z}\right)^{2}} d Q^{2} \int_{0}^{1} d \cos \xi \frac{1}{1024 \pi^{4}} \frac{l}{E^{3}} \\
& \times \sqrt{1-\frac{4 m_{H}^{2}}{Q^{2}}} F_{i}(\tau, Q, \xi) .
\end{aligned}
$$

Let us remind the reader that the total cross section is directly related to $\mathcal{F}_{1}(\tau)$,

$$
\sigma(\tau)=\frac{16}{3} \pi \mathcal{F}_{1}(\tau)
$$

We assume unpolarized $e^{+} e^{-}$beams and define observables as

$$
A_{i} \equiv \frac{\sum_{\tau} \mathcal{F}_{i}(\tau)}{\sum_{\tau} \mathcal{F}_{1}(\tau)}
$$

The symmetry property of $A_{i}$ is the same as that of the corresponding function $F_{i}$. We note the advantages of the observables $A_{i}$ :

(i) Some of systematic uncertainties such as the luminosity uncertainty cancel.

(ii) We expect that $\mathcal{F}_{i}(i=2,3, \ldots, 9)$ depend on the Higgs couplings in different ways from $\mathcal{F}_{1}$ (i.e. the total cross section) so that $\mathcal{F}_{i}(i=2,3, \ldots, 9)$ provide us different information on the Higgs couplings. The observables $A_{i}$ have the form that is sensitive to the difference between $\mathcal{F}_{i}$ $(i=2,3, \ldots, 9)$ and $\mathcal{F}_{1}$ in the dependence on the Higgs couplings.

For our numerical results, we set $E=500 \mathrm{GeV}$, $m_{Z}=91.188 \mathrm{GeV}, \quad \Gamma_{Z}=2.5 \mathrm{GeV}, \quad m_{H}=125.5 \mathrm{GeV}$, $\Gamma_{H}=0 \mathrm{GeV}$, and $e=\sqrt{4 \pi \alpha}$ with $\alpha=1 / 128 .{ }^{4}$ We assume that the $Z$ boson and the Higgs bosons can be reconstructed. The phase space integration is performed with the program BASES [41].

We numerically study the dependence of $A_{i}$ on the parameters in our effective Lagrangian. The single Higgs couplings to vector bosons such as $H Z Z$ may be precisely determined by measuring the polarization of the $Z$ boson in the process $e^{+} e^{-} \rightarrow Z H[39,42-44]$. Therefore, we focus

\footnotetext{
${ }^{4}$ The effect of a nonzero value for $\Gamma_{H}$ which is as small as the $\mathrm{SM}$ value is invisible in the following numerical results including the results in the right panel of Fig. 6.
} 

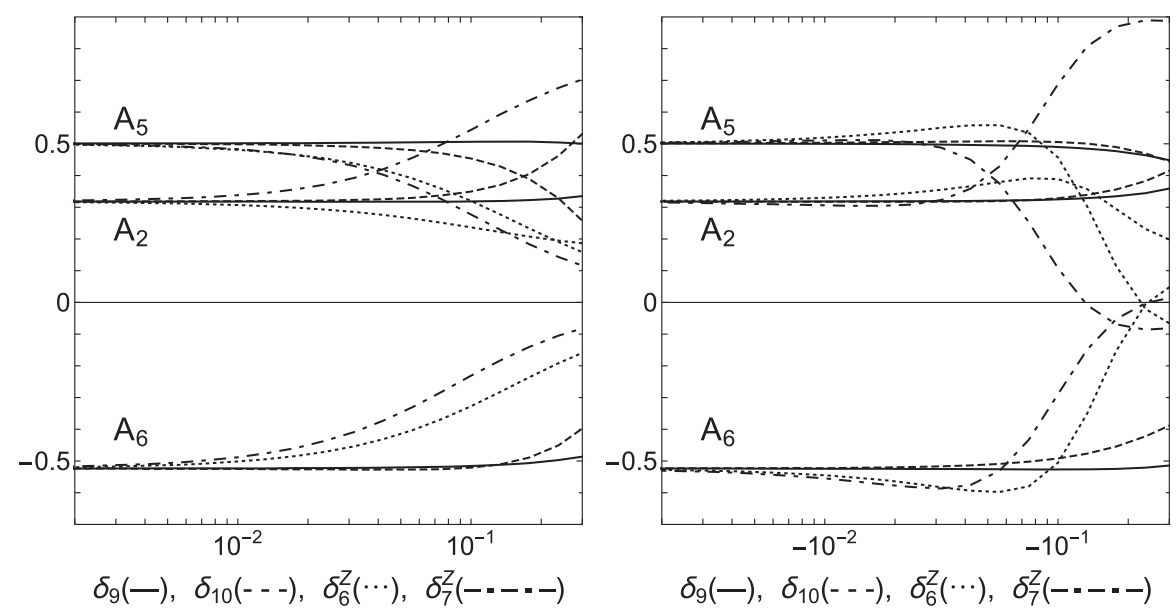

FIG. 5. $A_{2}, A_{5}$, and $A_{6}$ are shown as deviations caused by adding nonzero parameters $\delta_{9}$ (solid curve), $\delta_{10}$ (dashed curve), $\delta_{6}^{Z}$ (dotted curve), and $\delta_{7}^{Z}$ (broken curve). In the left panel the parameters take positive values, and in the right panel the parameters take negative values.

on the dependence on the parameters which cannot be accessed by single Higgs boson production processes. We choose as a benchmark point

$$
\delta_{2}^{Z}=\delta_{2}^{A}=\delta_{2}^{A A}=-\delta_{3}^{Z}=-\delta_{3}^{A}=-0.05
$$

The other parameters are set to zero. The total cross section with this choice is in agreement with the $\mathrm{SM}$ value within $10 \%$. In Fig. 5, the observables $A_{2}, A_{5}$, and $A_{6}$ are shown as deviations caused by adding nonzero parameters $\delta_{9}$ (solid curve), $\delta_{10}$ (dashed curve), $\delta_{6}^{Z}$ (dotted curve), and $\delta_{7}^{Z}$ (broken curve). In the left panel the parameters take positive values, and in the right panel the parameters take negative values. The results show that $A_{2,5,6}$ depend little on $\delta_{9}$. This indicates that $\mathcal{F}_{2,5,6}$ have the similar dependences on $\delta_{9}$ as $\mathcal{F}_{1}$. The results also show that $A_{2,5,6}$ depend largely on $\delta_{10}, \delta_{6}^{Z}$, and $\delta_{7}^{Z}$. This indicates that the observables $\mathcal{F}_{2,5,6}$ depend on these parameters in different ways from the total cross section. In the left panel of Fig. 6, the $C P$-odd observables $A_{8}$ and $A_{9}$ are shown as deviations caused by adding nonzero $C P$-odd parameters $\tilde{\delta}_{8}^{Z}$ (solid curve) and $\tilde{\delta}_{8}^{Z}=\tilde{\delta}_{8}^{A}$ (dashed curve). The results show that $A_{8,9}$ approach zero as the $C P$-odd parameters become small, as expected. These observables are nonzero only when $C P$ is violated. (Even if rescattering effects exist, these observables are identically zero as long as $C P$ is conserved.)

Because of the existence of the overall $\tau$ in the functions $F_{3}, F_{4}$, and $F_{7}$, the corresponding observables $A_{3}, A_{4}$, and $A_{7}$ can be suppressed. Longitudinally polarized $e^{+} e^{-}$ beams will be useful to study these three functions. We define observables as
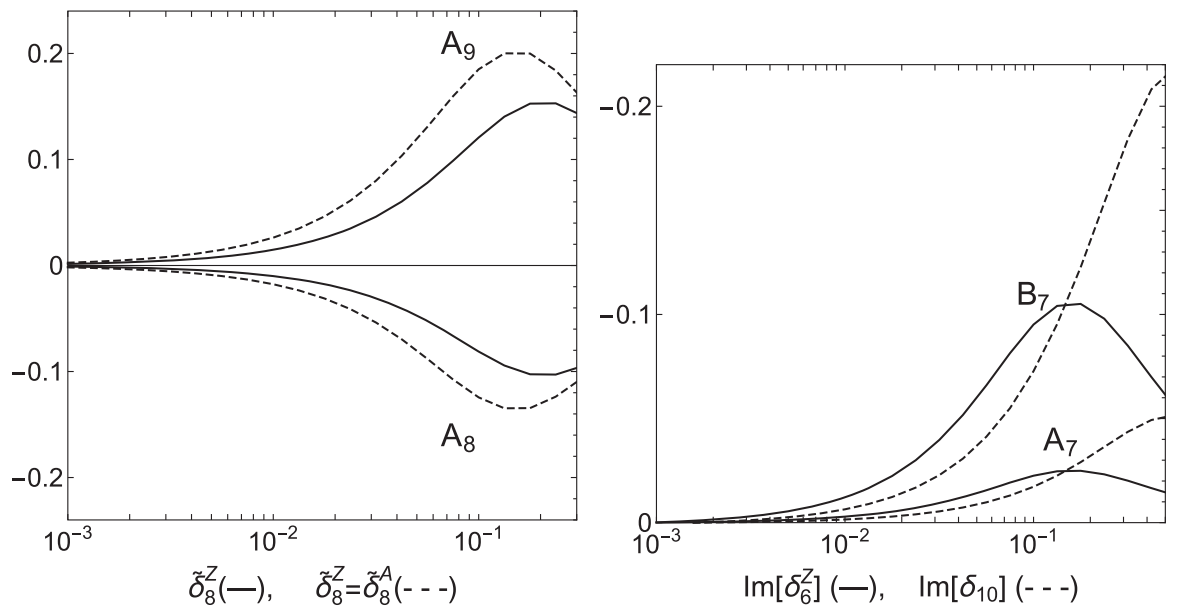

FIG. 6. Left: $C P$-odd observables $A_{8}$ and $A_{9}$ are shown as deviations caused by adding nonzero parameters $\tilde{\delta}_{8}^{Z}$ (solid curve) and $\tilde{\delta}_{8}^{Z}=\tilde{\delta}_{8}^{A}$ (dashed curve). Right: $C P \tilde{T}$-odd observables $A_{7}$ and $B_{7}$ are shown as deviations caused by adding nonzero imaginary parts in the parameters $\delta_{6}^{Z}$ (solid curve) and $\delta_{10}$ (dashed curve). 
$B_{i} \equiv \frac{\left(1+P_{-}\right)\left(1-P_{+}\right) \mathcal{F}_{i}(+)+\left(1-P_{-}\right)\left(1+P_{+}\right) \mathcal{F}_{i}(-)}{\left(1+P_{-}\right)\left(1-P_{+}\right) \mathcal{F}_{1}(+)+\left(1-P_{-}\right)\left(1+P_{+}\right) \mathcal{F}_{1}(-)}$,

where $P_{-}\left(-1 \leq P_{-} \leq 1\right)$ and $P_{+}\left(-1 \leq P_{+} \leq 1\right)$ denote the degrees of longitudinal polarization of the electron and the positron, respectively. We choose $\left(P_{-}, P_{+}\right)=$ $(-0.8,0.3)$ [1]. In the right panel of Fig. 6 , the $C P \tilde{T}$ odd observables $A_{7}$ and $B_{7}$ are shown as deviations caused by adding nonzero imaginary parts in the parameters $\delta_{6}^{Z}$ (solid curve) and $\delta_{10}$ (dashed curve). ${ }^{5}$ For the results in this panel, we choose as a benchmark point

$$
\delta_{2}^{Z}=\delta_{6}^{Z}=-\delta_{3}^{Z}=-\delta_{7}^{Z}=-\delta_{10}=-0.1,
$$

and the other parameters are set to zero. The total cross section with this choice is in agreement with the SM value within $30 \%$. The results show that $B_{7}>A_{7}$; i.e. the sensitivity to rescattering effects can be significantly increased by means of longitudinally polarized $e^{+} e^{-}$ beams. ${ }^{6}$ These observables are nonzero only when rescattering effects exist. (Even if $C P$ is violated, these observables are identically zero unless rescattering effects exist.) Note that the SM predictions in these $C P \tilde{T}$-odd observables are nonzero. The decay width in the $Z$ boson propagators contributes to these observables, since it indeed reflects the rescattering effect of the light fermions in the propagating $Z$ boson. The contribution is, however, negligibly small as we can observe in our results (i.e. $A_{7}$ and $B_{7}$ approach identically to zero as the imaginary part of $\delta_{6}^{Z}$ or that of $\delta_{10}$ becomes small).

\section{SUMMARY}

In this paper, we have derived the analytic expression for the differential cross section that in the most general case has the nine nonzero functions $F_{i}(i=1,2, \ldots, 9)$, for the process $e^{+} e^{-} \rightarrow Z H H$. The functions $F_{i}$ are the coefficients of the nine angular terms, depend on the Higgs couplings, and can be experimentally measured as observables. We have rederived the analytic forms of the observables which exist in the literature and have been widely used. We have found that all of these observables are directly related to $F_{1}$, which is just one of our nine functions. We have derived, for the first time, the analytic form of the $Z$ boson polar angle $\Theta$ distribution, which is related to three of our nine functions: $F_{1}, F_{2}$, and $F_{3}$. We have divided the nine functions into four categories under

\footnotetext{
${ }^{5}$ Rescattering effects can be approximately included by allowing imaginary parts in the Higgs couplings [36].

${ }^{6}$ This benefit from polarized beams, however, becomes less clear when $H Z \gamma, H \gamma \gamma$, and/or $H H Z \gamma$ couplings are turned on, due to the interference between the diagrams exchanging the $Z$ boson and those exchanging the photon.
}

$C P$ and $C P \tilde{T}$ : four even-even $\left(F_{1}, F_{2}, F_{5}, F_{6}\right)$, one even-odd $\left(F_{7}\right)$, two odd-even $\left(F_{8}, F_{9}\right)$, and two odd-odd $\left(F_{3}, F_{4}\right)$. This result is summarized in Table I.

We have introduced an effective Lagrangian for nonstandard Higgs couplings to the Higgs boson itself, the $Z$ boson, and the photon, and numerically studied the dependence of $\mathcal{F}_{i}$ [this is obtained by integrating $F_{i}$ over $Q^{2}$ and $\cos \xi$; see Eq. (5.3)] on the parameters in the effective Lagrangian. For this purpose, we have formed new observables $A_{i}$ and $B_{i}$ [Eqs. (5.5) and (5.7)] in terms of $\mathcal{F}_{i}$. These observables are defined in such a way that the differences between $\mathcal{F}_{i}(i=2,3, \ldots, 9)$ and $\mathcal{F}_{1}$ in the dependence on the Higgs couplings become apparent. Since $\mathcal{F}_{1}$ is directly related to the total cross section [Eq. (5.4)], by means of $A_{i}$ and $B_{i}$, we can learn whether $\mathcal{F}_{i}(i=2,3, \ldots, 9)$ provide us different information about the Higgs couplings than the total cross section. We have found that the three observables $\mathcal{F}_{2,5,6}$ have similar dependences on the constant shift of the trilinear self-coupling of the Higgs boson [i.e. $\delta_{9}$ in Eq. (5.1)] as the total cross section, while they have quite different dependences on the other $C P$-even parameters (i.e. $\delta_{10}, \delta_{6}^{Z}$, and $\delta_{7}^{Z}$ ) than the total cross section. This is shown in Fig. 5. The two $C P$-odd observables $\mathcal{F}_{8,9}$ and the $C P \tilde{T}$-odd observable $\mathcal{F}_{7}$ clearly have advantages over the total cross section in determining $C P$-odd parameters and in observing rescattering effects, respectively, since the total cross section is both $C P$-even and $C P \tilde{T}$-even. We have shown that the $C P$-odd observables $\mathcal{F}_{8,9}$ directly measure $C P$ violation, by showing that $\mathcal{F}_{8,9}$ approach identically to zero as the $C P$-odd parameters (i.e. $\tilde{\delta}_{8}^{Z}$ and $\tilde{\delta}_{8}^{A}$ ) become small. This is shown in the left panel of Fig. 6. Finally, we have shown that the use of longitudinally polarized $e^{+} e^{-}$beams can enhance the ability of the $C P \tilde{T}$-odd observable $\mathcal{F}_{7}$ which measures rescattering effects. This is shown in the right panel of Fig. 6.

\section{ACKNOWLEDGMENTS}

I sincerely appreciate the support from the Alexander von Humboldt Foundation.

\section{APPENDIX: THE EXPLICIT EXPRESSIONS OF THE FUNCTIONS $\boldsymbol{F}_{i}$}

In this appendix, we provide the explicit expressions of the functions $F_{i}$ in Eq. (3.1) in terms of the kinematic variables introduced in Sec. II and the effective Lagrangian parameters in Eq. (5.1). We write $F_{i}$ as

$$
F_{i}(\tau, Q, \xi)=\sum_{\lambda= \pm, 0} f_{i}(\tau, Q, \xi, \lambda) / v^{4},
$$

where $f_{i}$ are functions of the $Z$ boson helicity $\lambda$ in addition to $\tau, Q$, and $\xi$, and have the following forms: 
$f_{1}=\frac{1}{2}\left(|N(+, \lambda)|^{2}+|N(-, \lambda)|^{2}+|N(0, \lambda)|^{2}\right)$,

$f_{2}=\frac{1}{2}|N(0, \lambda)|^{2}$,

$f_{3}=\tau\left(|N(+, \lambda)|^{2}-|N(-, \lambda)|^{2}\right)$,

$f_{4}=\sqrt{2} \tau \operatorname{Re}\left[N(0, \lambda)^{*} N(+, \lambda)+N(-, \lambda)^{*} N(0, \lambda)\right]$,

$f_{5}=\frac{1}{\sqrt{2}} \operatorname{Re}\left[N(0, \lambda)^{*} N(+, \lambda)-N(-, \lambda)^{*} N(0, \lambda)\right]$,

$f_{6}=\operatorname{Re}\left[N(-, \lambda)^{*} N(+, \lambda)\right]$,

$f_{7}=\sqrt{2} \tau \operatorname{Im}\left[N(0, \lambda)^{*} N(+, \lambda)+N(-, \lambda)^{*} N(0, \lambda)\right]$,

$f_{8}=\frac{1}{\sqrt{2}} \operatorname{Im}\left[N(0, \lambda)^{*} N(+, \lambda)-N(-, \lambda)^{*} N(0, \lambda)\right]$,

$f_{9}=\operatorname{Im}\left[N(-, \lambda)^{*} N(+, \lambda)\right]$.

The functions $N(\sigma, \lambda)$ in the above equations receive contributions from the Feynman diagrams shown in Fig. 2. The contribution from diagram (1) of Fig. 2, in which the intermediate vector boson $V$ is the $Z$ boson $(V=Z)$ or the photon $(V=A)$, is given by

$$
\begin{aligned}
N_{1}^{V}(\sigma= \pm, \lambda= \pm)= & g_{\tau}^{V} E \sigma \delta_{\sigma \lambda} D_{V}(p) D_{H}(q) \\
& \times\left[3 m_{H}^{2}\left(1+\delta_{9}\right)-\delta_{10}\left(2 m_{H}^{2}+Q^{2}\right)\right] \\
& \times\left(a+b E w-c E^{2}-d m_{Z}^{2}+i \lambda \tilde{e} E l\right), \\
N_{1}^{V}(\sigma=0, \lambda= \pm)= & 0, \quad N_{1}^{V}(\sigma= \pm, \lambda=0)=0, \\
N_{1}^{V}(\sigma=0, \lambda=0)= & -g_{\tau}^{V} E D_{V}(p) D_{H}(q) \\
& \times\left[3 m_{H}^{2}\left(1+\delta_{9}\right)-\delta_{10}\left(2 m_{H}^{2}+Q^{2}\right)\right] \\
& \times\left[\left(a-c E^{2}-d m_{Z}^{2}\right) w+b E m_{Z}^{2}\right] / m_{Z},
\end{aligned}
$$

where the couplings $g_{\tau}^{V}$ are

$$
\begin{aligned}
& g_{+}^{Z}=\frac{2 m_{Z}}{v} \sin ^{2} \theta_{w}, \quad g_{-}^{Z}=\frac{2 m_{Z}}{v}\left(-0.5+\sin ^{2} \theta_{w}\right), \\
& g_{+}^{A}=g_{-}^{A}=-e,
\end{aligned}
$$

and

$$
D_{i}(p)=\left(p \cdot p-m_{i}^{2}+i m_{i} \Gamma_{i}\right)^{-1}
$$

denotes the propagator factor for a particle $i$ with its four-momentum $p$, mass $m_{i}$, and decay width $\Gamma_{i}$. The coefficients $a, b, c, d, \tilde{e}$ are written in terms of the effective Lagrangian parameters as follows: When $V=Z$,

$$
\begin{aligned}
& a=2 m_{Z}^{2}\left(1+\delta_{1}\right), \quad b=4\left(\delta_{2}^{Z}-\delta_{3}^{Z}\right), \\
& c=d=-2 \delta_{3}^{Z}, \quad \tilde{e}=-4 \tilde{\delta}_{4}^{Z} .
\end{aligned}
$$

When $V=A$,

$a=0, \quad b=2\left(\delta_{2}^{A}-\delta_{3}^{A}\right), \quad c=-2 \delta_{3}^{A}$,

$d=0, \quad \tilde{e}=-2 \tilde{\delta}_{4}^{A}$.

The contribution from diagram (2) of Fig. 2, in which the intermediate vector boson $V_{1}(=Z, A)$ has the fourmomentum $p^{\mu}$ and $V_{2}(=Z, A)$ has the four-momentum $t^{\mu}$, is given by

$$
\begin{aligned}
N_{2}^{V_{1} V_{2}}(\sigma= \pm, \lambda= \pm)= & g_{\tau}^{V_{1}} E \sigma D_{V_{1}}(p) D_{V_{2}}(t)\left\{\delta_{\sigma \lambda}\left[\bar{a}_{1} \bar{a}_{2}+\tilde{e}_{1} \tilde{e}_{2} E t^{3}\left(l t^{0}+w t^{3}\right)+i \lambda \bar{a}_{1} \tilde{e}_{2}\left(l t^{0}+w t^{3}\right)-i \lambda \tilde{e}_{1} \bar{a}_{2} E t^{3}\right]\right. \\
& \left.-\frac{1}{2} r^{2} \sin ^{2} \xi\left(X_{t}+i \sigma \tilde{e}_{1} b_{2} E l+i \lambda b_{1} \tilde{e}_{2} E l-\sigma \lambda \tilde{e}_{1} \tilde{e}_{2} E w\right)\right\} \\
N_{2}^{V_{1} V_{2}}(\sigma=0, \lambda= \pm)= & -\frac{1}{\sqrt{2}} g_{\tau}^{V_{1}} E D_{V_{1}}(p) D_{V_{2}}(t) r \sin \xi\left[\bar{a}_{1} b_{2} l+t^{3} X_{t}+\tilde{e}_{1} \tilde{e}_{2} E\left(l t^{0}+w t^{3}\right)\right. \\
& \left.+i \lambda\left(\bar{a}_{1} \tilde{e}_{2} w+b_{1} \tilde{e}_{2} t^{3} E l-\tilde{e}_{1} \bar{a}_{2} E\right)\right] \\
N_{2}^{V_{1} V_{2}}(\sigma= \pm, \lambda=0)= & \frac{1}{\sqrt{2}} g_{\tau}^{V_{1}} E \sigma D_{V_{1}}(p) D_{V_{2}}(t) r \sin \xi\left\{\left(l t^{0}+w t^{3}\right) X_{t}-b_{1} \bar{a}_{2} E l+\tilde{e}_{1} \tilde{e}_{2} E t^{3} m_{Z}^{2}\right. \\
& \left.+i \sigma\left[\bar{a}_{1} \tilde{e}_{2} m_{Z}^{2}-\tilde{e}_{1} \bar{a}_{2} E w+\tilde{e}_{1} b_{2}\left(l t^{0}+w t^{3}\right) E l\right]\right\} / m_{Z}, \\
N_{2}^{V_{1} V_{2}}(\sigma=0, \lambda=0)= & -g_{\tau}^{V_{1}} E D_{V_{1}}(p) D_{V_{2}}(t)\left[\bar{a}_{1} \bar{a}_{2} w-\bar{a}_{1} b_{2} l\left(l t^{0}+w t^{3}\right)-t^{3}\left(l t^{0}+w t^{3}\right) X_{t}\right. \\
& \left.+b_{1} \bar{a}_{2} t^{3} E l+\tilde{e}_{1} \tilde{e}_{2} E m_{Z}^{2} r^{2} \sin ^{2} \xi\right] / m_{Z},
\end{aligned}
$$


where

$$
\begin{aligned}
X_{t}= & \bar{a}_{1} c_{2}+b_{1} b_{2} E w-b_{1} c_{2} E t^{0}+d_{1} \bar{a}_{2}-d_{1} b_{2}\left(w t^{0}+l t^{3}\right) \\
& +d_{1} c_{2} t \cdot t-\eta \frac{1}{m_{Z}^{2}}\left(\bar{a}_{1}-b_{1} E t^{0}+d_{1} t \cdot t\right) \\
& \times\left[\bar{a}_{2}-b_{2}\left(w t^{0}+l t^{3}\right)+c_{2} t \cdot t\right] .
\end{aligned}
$$

Let us remind the reader that the $\mu=0,3$ components of $t^{\mu}$ (i.e. $t^{0}$ and $t^{3}$ ) which appear above do not depend on $\phi$; see Eq. (2.4). The coefficients $\bar{a}_{1,2}$ are given by

$$
\begin{aligned}
& \bar{a}_{1}=a_{1}+b_{1} E t^{0}-c_{1} E^{2}-d_{1} t \cdot t, \\
& \bar{a}_{2}=a_{2}+b_{2}\left(w t^{0}+l t^{3}\right)-c_{2} t \cdot t-d_{2} m_{Z}^{2} .
\end{aligned}
$$

The coefficients $a_{i}, b_{i}, c_{i}, d_{i}, \tilde{e}_{i}(i=1,2)$ and the parameter $\eta$ in Eq. (A8) take the following values: When $\left(V_{1}, V_{2}\right)=(Z, Z)$,

$$
\begin{array}{ll}
a_{1}=a_{2}=2 m_{Z}^{2}\left(1+\delta_{1}\right), & b_{1}=b_{2}=4\left(\delta_{2}^{Z}-\delta_{3}^{Z}\right), \\
c_{1}=d_{1}=c_{2}=d_{2}=-2 \delta_{3}^{Z}, & \tilde{e}_{1}=\tilde{e}_{2}=-4 \tilde{\delta}_{4}^{Z}, \quad \eta=1 .
\end{array}
$$

When $\left(V_{1}, V_{2}\right)=(A, Z)$,

$$
\begin{aligned}
& a_{1}=0, \quad b_{1}=2\left(\delta_{2}^{A}-\delta_{3}^{A}\right), \quad c_{1}=-2 \delta_{3}^{A}, \\
& d_{1}=0, \quad \tilde{e}_{1}=-2 \tilde{\delta}_{4}^{A}, \\
& a_{2}=2 m_{Z}^{2}\left(1+\delta_{1}\right), \quad b_{2}=4\left(\delta_{2}^{Z}-\delta_{3}^{Z}\right), \\
& c_{2}=d_{2}=-2 \delta_{3}^{Z}, \quad \tilde{e}_{2}=-4 \tilde{\delta}_{4}^{Z}, \quad \eta=1 .
\end{aligned}
$$

When $\left(V_{1}, V_{2}\right)=(Z, A)$,

$$
\begin{aligned}
& a_{1}=0, \quad b_{1}=2\left(\delta_{2}^{A}-\delta_{3}^{A}\right), \quad c_{1}=0, \\
& d_{1}=-2 \delta_{3}^{A}, \quad \tilde{e}_{1}=-2 \tilde{\delta}_{4}^{A}, \\
& a_{2}=0, \quad b_{2}=2\left(\delta_{2}^{A}-\delta_{3}^{A}\right), \quad c_{2}=-2 \delta_{3}^{A}, \\
& d_{2}=0, \quad \tilde{e}_{2}=-2 \tilde{\delta}_{4}^{A}, \quad \eta=0 .
\end{aligned}
$$

When $\left(V_{1}, V_{2}\right)=(A, A)$,

$$
\begin{array}{ll}
a_{1}=0, & b_{1}=4 \delta_{2}^{A A}, \quad c_{1}=0, \\
d_{1}=0, & \tilde{e}_{1}=-4 \tilde{\delta}_{4}^{A A}, \\
a_{2}=0, & b_{2}=2\left(\delta_{2}^{A}-\delta_{3}^{A}\right), \quad c_{2}=-2 \delta_{3}^{A}, \\
d_{2}=0, & \tilde{e}_{2}=-2 \tilde{\delta}_{4}^{A}, \quad \eta=0 .
\end{array}
$$

The contribution from diagram (3) of Fig. 2, in which the intermediate vector boson $V_{1}(=Z, A)$ has the fourmomentum $p^{\mu}$ and $V_{2}(=Z, A)$ has the four-momentum $u^{\mu}$, can be obtained by the simple replacement $t^{\mu} \rightarrow u^{\mu}$ in Eq. (A7),

$$
\begin{gathered}
N_{3}^{V_{1} V_{2}}(\sigma= \pm, \lambda= \pm)=\left.N_{2}^{V_{1} V_{2}}(\sigma= \pm, \lambda= \pm)\right|_{t^{\mu} \rightarrow u^{\mu}}, \\
N_{3}^{V_{1} V_{2}}(\sigma=0, \lambda= \pm)=-\left.N_{2}^{V_{1} V_{2}}(\sigma=0, \lambda= \pm)\right|_{t^{\mu} \rightarrow u^{\mu}}, \\
N_{3}^{V_{1} V_{2}}(\sigma= \pm, \lambda=0)=-\left.N_{2}^{V_{1} V_{2}}(\sigma= \pm, \lambda=0)\right|_{t^{\mu} \rightarrow u^{\mu}}, \\
N_{3}^{V_{1} V_{2}}(\sigma=0, \lambda=0)=\left.N_{2}^{V_{1} V_{2}}(\sigma=0, \lambda=0)\right|_{t^{\mu} \rightarrow u^{\mu}},
\end{gathered}
$$

where the minus sign in the second and the third equations originates from the existence of the overall $r$ [the replacement $t^{\mu} \rightarrow u^{\mu}$ can be translated into $r \rightarrow-r$; see Eq. (2.4)]. The replacement $t^{\mu} \rightarrow u^{\mu}$ must be performed in Eqs. (A8) and (A9), too. The coefficients $a_{i}, b_{i}, c_{i}, d_{i}, \tilde{e}_{i}(i=1,2)$ and the parameter $\eta$ take the same values as Eq. (A10). The contribution from diagram (4) of Fig. 2, in which the intermediate vector boson $V$ is the $Z$ boson $(V=Z)$ or the photon $(V=A)$, is given by

$$
\begin{aligned}
N_{4}^{V}(\sigma= \pm, \lambda= \pm)= & g_{\tau}^{V} E \sigma \delta_{\sigma \lambda} D_{V}(p)\left(a+b E w-c E^{2}\right. \\
& \left.-d m_{Z}^{2}+i \lambda \tilde{e} E l\right) \\
N_{4}^{V}(\sigma=0, \lambda= \pm)= & 0, \quad N_{4}^{V}(\sigma= \pm, \lambda=0)=0 \\
N_{4}^{V}(\sigma=0, \lambda=0)= & -g_{\tau}^{V} E D_{V}(p) \\
& \times\left[\left(a-c E^{2}-d m_{Z}^{2}\right) w+b E m_{Z}^{2}\right] / m_{Z} .
\end{aligned}
$$

The coefficients $a, b, c, d, \tilde{e}$ are written as follows: When $V=Z$,

$$
\begin{aligned}
& a=2 m_{Z}^{2}\left(1+\delta_{5}\right), \quad b=4\left(\delta_{6}^{Z}-\delta_{7}^{Z}\right), \\
& c=d=-2 \delta_{7}^{Z}, \quad \tilde{e}=-4 \tilde{\delta}_{8}^{Z} .
\end{aligned}
$$

When $V=A$,

$a=0, \quad b=2\left(\delta_{6}^{A}-\delta_{7}^{A}\right), \quad c=-2 \delta_{7}^{A}$,

$d=0, \quad \tilde{e}=-2 \tilde{\delta}_{8}^{A}$.

The sum of all the functions in Eqs. (A3), (A7), (A11), and (A12) provides the functions $N(\sigma, \lambda)$ in Eq. (A2),

$$
\begin{aligned}
N(\sigma, \lambda)= & N_{1}^{Z}(\sigma, \lambda)+N_{1}^{A}(\sigma, \lambda)+N_{2}^{Z Z}(\sigma, \lambda)+N_{2}^{A Z}(\sigma, \lambda) \\
& +N_{2}^{Z A}(\sigma, \lambda)+N_{2}^{A A}(\sigma, \lambda)+N_{3}^{Z Z}(\sigma, \lambda) \\
& +N_{3}^{A Z}(\sigma, \lambda)+N_{3}^{Z A}(\sigma, \lambda)+N_{3}^{A A}(\sigma, \lambda) \\
& +N_{4}^{Z}(\sigma, \lambda)+N_{4}^{A}(\sigma, \lambda) .
\end{aligned}
$$


[1] H. Baer et al., The International Linear Collider Technical Design Report-Volume 2: Physics, arXiv:1306.6352.

[2] D. M. Asner et al., ILC Higgs White Paper, arXiv:1310 .0763 .

[3] A. Arbey et al., Physics at the $e^{+} e^{-}$linear collider, Eur. Phys. J. C 75, 371 (2015).

[4] K. Fujii et al., Physics case for the international linear collider, arXiv:1506.05992.

[5] L. Linssen, A. Miyamoto, M. Stanitzki, and H. Weerts, Physics and detectors at CLIC: CLIC conceptual design report, arXiv:1202.5940.

[6] V. A. Ilyin, A. E. Pukhov, Y. Kurihara, Y. Shimizu, and T. Kaneko, Probing the $H^{3}$ vertex in $e^{+} e^{-}, \gamma e$ and $\gamma \gamma$ collisions for light and intermediate Higgs bosons, Phys. Rev. D 54, 6717 (1996).

[7] F. Boudjema and E. Chopin, Double Higgs production at the linear colliders and the probing of the Higgs self-coupling, Z. Phys. C 73, 85 (1996).

[8] D. J. Miller and S. Moretti, Can the trilinear Higgs selfcoupling be measured at future linear colliders?, Eur. Phys. J. C 13, 459 (2000).

[9] M. Battaglia, E. Boos, and W.-M. Yao, Studying the Higgs potential at the $e^{+} e^{-}$linear collider, eConf C010630, E3016 (2001).

[10] C. Castanier, P. Gay, P. Lutz, and J. Orloff, Higgs selfcoupling measurement in $e^{+} e^{-}$collisions at center-of-mass energy of $500 \mathrm{GeV}$, arXiv:hep-ex/0101028.

[11] Y. Yasui et al., Measurement of the Higgs self-coupling at JLC, arXiv:hep-ph/0211047.

[12] Y. Takubo, Analysis of ZHH in the 4-jet mode, arXiv: 0901.3598

[13] M. Faucci Giannelli, Sensitivity to the Higgs self-coupling using the ZHH channel, arXiv:0901.4895.

[14] U. Baur, Measuring the Higgs boson self-coupling at high energy $e^{+} e^{-}$colliders, Phys. Rev. D 80, 013012 (2009).

[15] J. Tian, Study of Higgs self-coupling at the ILC based on the full detector simulation at $\mathrm{s}=500 \mathrm{GeV}$ and $\mathrm{s}=1 \mathrm{TeV}$, in Helmholtz Alliance Linear Collider Forum: Proceedings of the Workshops Hamburg, Munich, Germany (DESY, Hamburg, 2013), pp. 224-247.

[16] J. Strube (ILC Physics and Detector Study Collaboration), Measurement of the Higgs boson coupling to the top quark and the Higgs boson self-coupling at the ILC, Nucl. Part. Phys. Proc. 273-275, 2463 (2016).

[17] G. Aad et al. (ATLAS Collaboration), Observation of a new particle in the search for the Standard Model Higgs boson with the ATLAS detector at the LHC, Phys. Lett. B 716, 1 (2012).

[18] S. Chatrchyan et al. (CMS Collaboration), Observation of a new boson at a mass of $125 \mathrm{GeV}$ with the CMS experiment at the LHC, Phys. Lett. B 716, 30 (2012).

[19] G. J. Gounaris, D. Schildknecht, and F. M. Renard, Test of Higgs boson nature in $e^{+} e^{-} \rightarrow H H Z$, Phys. Lett. 83B, 191 (1979).

[20] V. D. Barger, T. Han, and R. J. N. Phillips, Double Higgs boson bremsstrahlung from $W$ and $Z$ bosons at supercolliders, Phys. Rev. D 38, 2766 (1988).

[21] Q.-H. Dai, W.-G. Ma, and Y.-Y. Liu, Test of Higgs couplings in $e+e-$ colliders, Phys. Lett. B 226, 180 (1989).
[22] R.-Y. Zhang, W.-G. Ma, H. Chen, Y.-B. Sun, and H.-S. Hou, Full $\mathrm{O}\left(\alpha_{e w}\right)$ electroweak corrections to $e^{+} e^{-} \rightarrow H H Z$, Phys. Lett. B 578, 349 (2004).

[23] G. Belanger, F. Boudjema, J. Fujimoto, T. Ishikawa, T. Kaneko, Y. Kurihara, K. Kato, and Y. Shimizu, Full $\mathrm{O}(\alpha)$ electroweak corrections to double Higgs strahlung at the linear collider, Phys. Lett. B 576, 152 (2003).

[24] J.-i. Kamoshita, Y. Okada, M. Tanaka, and I. Watanabe, Studying the Higgs potential via $e^{+} e^{-} \rightarrow Z h h$, arXiv:hep$\mathrm{ph} / 9602224$.

[25] A. Djouadi, H. E. Haber, and P. M. Zerwas, Multiple production of MSSM neutral Higgs bosons at high-energy $e^{+} e^{-}$colliders, Phys. Lett. B 375, 203 (1996).

[26] P. Osland and P. N. Pandita, Measuring the trilinear couplings of MSSM neutral Higgs bosons at high-energy $e^{+} e^{-}$ colliders, Phys. Rev. D 59, 055013 (1999).

[27] A. Djouadi, W. Kilian, M. Mühlleitner, and P. M. Zerwas, Testing Higgs self-couplings at $e^{+} e^{-}$linear colliders, Eur. Phys. J. C 10, 27 (1999).

[28] A. Djouadi, The Higgs sector of supersymmetric theories and the implications for high-energy colliders, Eur. Phys. J. C 59, 389 (2009).

[29] R. Gröber and M. Mühlleitner, Higgs pair production in composite Higgs models at the ILC, in Helmholtz Alliance Linear Collider Forum: Proceedings of the Workshops Hamburg, Munich, Germany (DESY, Hamburg, 2013), pp. 352-363.

[30] S. Kanemura, K. Kaneta, N. Machida, S. Odori, and T. Shindou, Single and double production of the Higgs boson at hadron and lepton colliders in minimal composite Higgs models, Phys. Rev. D 94, 015028 (2016).

[31] E. Asakawa, D. Harada, S. Kanemura, Y. Okada, and K. Tsumura, Higgs boson pair production in new physics models at hadron, lepton, and photon colliders, Phys. Rev. D 82, 115002 (2010).

[32] V. Barger, T. Han, P. Langacker, B. McElrath, and P. Zerwas, Effects of genuine dimension-six Higgs operators, Phys. Rev. D 67, 115001 (2003).

[33] R. Contino, C. Grojean, D. Pappadopulo, R. Rattazzi, and A. Thamm, Strong Higgs interactions at a linear collider, J. High Energy Phys. 02 (2014) 006.

[34] S. Kumar and P. Poulose, Influence of anomalous VVH and VVHH on determination of Higgs self-couplings at ILC, arXiv:1408.3563.

[35] T. Barklow, K. Fujii, S. Jung, M. E. Peskin, and J. Tian, Model-independent determination of the triple Higgs coupling at $e^{+} e^{-}$colliders, arXiv:1708.09079.

[36] K. Hagiwara, R. D. Peccei, D. Zeppenfeld, and K. Hikasa, Probing the weak boson sector in $e^{+} e^{-} \rightarrow W^{+} W^{-}$, Nucl. Phys. B282, 253 (1987).

[37] D. Binosi and L. Theussl, JaxoDraw: A graphical user interface for drawing Feynman diagrams, Comput. Phys. Commun. 161, 76 (2004).

[38] J. Nakamura, Polarisations of the $Z$ and $W$ bosons in the processes $p p \rightarrow Z H$ and $p p \rightarrow W^{ \pm} H$, J. High Energy Phys. 08 (2017) 008.

[39] K. Hagiwara and M. L. Stong, Probing the scalar sector in $e^{+} e^{-} \rightarrow f \bar{f} H$, Z. Phys. C 62, 99 (1994). 
[40] K. Hagiwara, S. Ishihara, R. Szalapski, and D. Zeppenfeld, Low-energy effects of new interactions in the electroweak boson sector, Phys. Rev. D 48, 2182 (1993).

[41] S. Kawabata, A New version of the multidimensional integration and event generation package BASES/SPRING, Comput. Phys. Commun. 88, 309 (1995).
[42] R. Rattazzi, Anomalous interactions at the $Z^{0}$ pole, Z. Phys. C 40, 605 (1988).

[43] V. D. Barger, K.-m. Cheung, A. Djouadi, B. A. Kniehl, and P. M. Zerwas, Higgs bosons: Intermediate mass range at $e^{+} e^{-}$colliders, Phys. Rev. D 49, 79 (1994).

[44] K. Hagiwara, S. Ishihara, J. Kamoshita, and B. A. Kniehl, Prospects of measuring general Higgs couplings at $e^{+} e^{-}$ linear colliders, Eur. Phys. J. C 14, 457 (2000). 